\title{
Hemodynamics in the Aorta and Pulmonary Arteries of Congenital Heart Disease Patients: A Mini Review
}

\author{
Lauren Johnston, Maria Boumpouli, Asimina Kazakidi*
}

Department of Biomedical Engineering, University of Strathclyde, Glasgow, UK

\section{Article Info}

\section{Article Notes}

Received: May 2, 2021

Accepted: June 18, 2021

\section{${ }^{*}$ Correspondence:}

Dr. Asimina Kazakidi, Department of Biomedical

Engineering, University of Strathclyde, Glasgow, UK; Email: asimina.kazakidi@strath.ac.uk.

( 2021 Kazakidi A. This article is distributed under the terms of the Creative Commons Attribution 4.0 International License.

\section{Keywords:}

Hemodynamics

Computational fluid dynamics

Cardiovascular disease

Congenital heart disease

Aorta

Pulmonary artery

\section{Abstract}

Congenital heart disease, which affects more than one million newborns globally each year, contributes to an increased risk of cardiovascular disease and ultimately reduced life expectancy. Computational fluid dynamics (CFD) enables detailed, non-invasive characterization of complex physiological pressure and flow fields, thus improving our understanding of congenital heart disease hemodynamics.

In recent years, this has driven clinical decision-making, surgical planning, and the evaluation of innovative surgical techniques. In this mini review, CFD methods applied to the study of congenital abnormalities, with a focus on the aorta and pulmonary bifurcation, are discussed. The clinical relevance and future directions of CFD modelling are also reviewed.

\section{Congenital Abnormalities of the Aorta and Pulmonary Bifurcation}

Globally, an estimated 1.35 million newborns are diagnosed each year with congenital heart disease (CHD) ${ }^{1}$, defined as structural abnormalities of the heart, and/or great vessels, which are present from birth. The American Heart Association defines 21 malformations in the category of $\mathrm{CHD}^{2}$, ranging from minor lesions, such as bicuspid aortic valve (BAV), to severe, such as coarctation of the aorta (CoA) and tetralogy of Fallot (TOF). Congenital abnormalities of the aorta and pulmonary bifurcation contribute to an increased risk of cardiovascular disease and ultimately reduced life expectancy ${ }^{3-4}$.

Approximately 8\% of CHD patients have coarctation of the aorta ${ }^{5}$, defined as a narrowing of the vessel which leads to an obstruction of blood flow. If symptomatic, surgical intervention is required to widen the aortic segment and long-term monitoring is essential due to the risk of hypertension and aneurysm formation. Furthermore, Tetralogy of Fallot, the most common cyanotic congenital heart disease, accounts for $6.1 \%$ of $\mathrm{CHDs}^{5}$. TOF patients are diagnosed with four cardiac defects: right ventricular hypertrophy, pulmonary stenosis, overriding aorta and ventricular septal defect, which require surgical intervention early in childhood. However, these patients are still at risk of needing further surgical treatment, particularly if pulmonary regurgitation and branch pulmonary obstruction are present $t^{6}$. The optimal timing for clinicians to treat pulmonary regurgitation and branch pulmonary obstruction is a challenge and requires improved understanding of the hemodynamic alterations in the pulmonary arterial bifurcation. In a recent publication by Boumpouli et al., $2020^{7}$ (with title "Blood flow simulations in the pulmonary bifurcation in relation to adult patients with repaired 
tetralogy of Fallot", Med Eng Phys 85:123-138), the underlying blood flow characteristics in the pulmonary trunk were studied using computational fluid dynamics methods ${ }^{7}$.

\section{Computational Fluid Dynamics (CFD)}

Blood flow simulations, using patient-specific geometries and boundary conditions, can improve our understanding of the underlying hemodynamics, strengthen the diagnostic potential, and enhance the possibility for personalized patient-specific medicine. This non-invasive method enables detailed characterization of complex physiological pressure and flow fields, and the computation of variables not measurable in-vivo. Increasingly complex models of arterial vessels have deepened our knowledge of the relationship between anatomical and hemodynamic factors related to the initiation and progression of cardiovascular diseases. Patient-specific and multiscale models have enhanced prognostic capabilities and progressed towards customization in patient treatment. It is, therefore, unsurprising that computational fluid dynamics is increasingly applied to the study of CHD ${ }^{8-11}$.

A computational fluid dynamic workflow can be broadly divided into three general steps: pre-processing (where the fluid domain is defined and discretized), processing (where the fluid problem is numerically solved), and postprocessing (where the problem solution is visualized and analyzed) (Figure $1^{12}$ details for the MR images can be found $\mathrm{in}^{13}$ ). For hemodynamics problems, the specific details of each step can be performed with a varying degree of patient-specificity, physiological accuracy and numerical complexity. In the pre-processing stage, the fluid domain can be one- (1D), two- (2D) or three-dimensional (3D), in the case of simplified geometries; zero-dimensional (0D, lumped-parameter models), assuming the circulatory system analogous to electric circuits to capture peripheral resistance, arterial compliance, impedance, and inertance ${ }^{14}$; reconstructed from clinical images, in the case of patient-specific geometries; or coupled, for example combining 0D with 3D flow analysis or with a solid domain for fluid-structure interaction. Although simplified, lower-order models can provide information about the general characteristics of the flow, including velocity and pressure, while also facilitate the investigation of various morphological parameters and boundary conditions ${ }^{7}$.

During the discretization process in body-conforming methods, the fluid domain is subdivided into a finite number of subdomains, depending on the numerical approximation scheme. The fluid problem is, in turn, further defined by assigning appropriate initial and boundary conditions (BCs) at the model boundaries (inlets, outlets, walls, periodic and symmetry faces etc.). For physiological flows, the inlet and outlet BCs can include quasi-steady or transient pulsatile flows, obtained from the literature or patient clinical measurements using Doppler ultrasonography and/or magnetic resonance imaging ${ }^{11}$.

Physiologically-correct time-dependent and/or time and space varying velocity profiles can be extracted and assigned at the boundaries of the models ${ }^{15}$. Unless modelling highly compliant vessels or the contraction of the heart, the interface between the vessel and lumen (fluid domain wall) can be assumed rigid for large vessels such as the aorta and pulmonary bifurcation, as the overall characteristics of the flow are not affected ${ }^{16}$.

In the processing stage, the continuous fluid problem is solved using the incompressible Navier-Stokes equations which govern the flow of viscous Newtonian or nonNewtonian fluids ${ }^{7}$. The resulting fluid solution from a CFD analysis provides detailed information on fundamental quantities, such as velocity and pressure fields (including 3D streamlines, 2D slices, 2D vector-fields etc.), from which other secondary flow parameters can be calculated at the post-processing stage, at every discrete position. The fluid solution can, therefore, be visualized through additional hemodynamic parameters, such as Wall Shear Stress (WSS), oscillatory shear index, relative residence time, etc., which are useful to identify areas where flow departs from a laminar, unidirectional pattern, and are commonly used in cardiovascular modelling.

\section{CFD Studies on CHD Hemodynamics}

Several groups have studied the hemodynamics behind a variety of congenital cardiovascular malformations ${ }^{9,17,18}$. Computational models of bicuspid aortic valve, which accounts for greater morbidity and mortality than all other

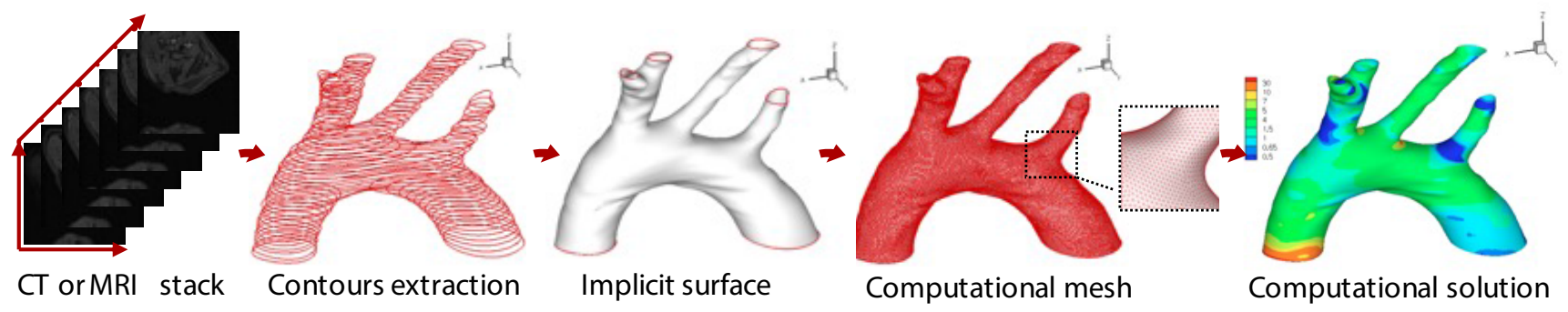

Figure 1. Subject-specific computational fluid dynamic workflow for the aortic arch ${ }^{12}$. 
congenital heart diseases combined, revealed turbulent jet flow through the valve and high shear stress on the aortic wall $^{19,20}$. In a computational analysis of a BAV patient, conducted by the authors, highly disturbed flow was found in the ascending aorta, and elevated, heterogeneous wall shear stress patterns at the inner and outer walls of the descending aorta ${ }^{18}$. Flow characteristics such as the Reynolds number (Re), branch flow division and reverse flow, affect the wall shear stress patterns in the descending thoracic aorta, where increasing Re results in lower wall shear stress at the lateral sides of branch ostia ${ }^{21,22}$. The impaired hemodynamics in CHD patients can lead to secondary pathologies, including aortic stenosis, dilatation, and aneurysms. In CHD patients with coarctation of the aorta, computational studies have revealed significantly altered flow dynamics, with a pressure-drop across the coarctation site, and a stenotic velocity jet resulting in elevated wall shear stress downstream ${ }^{23,24}$. We reported similar findings in a CoA patient ${ }^{18}$.

Several authors have successfully reported the use of MRI-based CFD as an alternative to invasive diagnostic catheterization in coarctation of the aorta ${ }^{25,26}$. In 2015, Cosentino et al. ${ }^{27}$ published a case study on the use of patient-specific modelling to optimise the intervention in an individual with complex re-coarctation of the aorta and Capelli et al. ${ }^{9}$ utilized CFD simulations to optimise the sizing and positioning of CoA stenting. Szopos et al. ${ }^{28}$ suggested increased surveillance of "gothic" arch patients with coarctation repair after evaluating fluid-wall interactions using CFD.

Computational investigations of endovascular coarctation stent repair found negligible increases in cardiac work when compared to open surgical repair, and a greater percentage of low TAWSS along the anterior wall of the descending aorta ${ }^{29}$.

Computational methods have been utilized in various studies in order to understand the hemodynamic environment in the pulmonary bifurcation of healthy and diseased populations. The pulmonary junction is found hemodynamically efficient in healthy subjects ${ }^{30}$, with a $10 \%$ decrease in energy efficiency under exercise conditions $^{31}$. In patients with TOF, decreasing the angle of the left pulmonary artery results in an increase of energy $\operatorname{loss}^{32}$. Flow recirculation occurs in the left pulmonary artery (LPA) branch in both healthy and TOF patients ${ }^{7,30,32}$, which is also the branch where regurgitation appears first in the repaired TOF population ${ }^{33}$. Branch stenosis, commonly observed in the LPA branch of TOF patients, can also alter the flow environment by displacing the point where flow impinges, a critical location in the wall shear stress distribution and differential branch pressure ${ }^{7,32,34}$, while also promoting an asymmetrical flow split in the two branches ${ }^{35}$. In addition, vortices appear in stenotic regions of the pulmonary branches, compared to the helical flow which is visible in non-stenotic branches ${ }^{35}$. Comparison of wall shear stress patterns between healthy and diseased populations has revealed that WSS is significantly lower in the proximal pulmonary arterial branches, in the case of pulmonary hypertension ${ }^{31}$.

\section{Clinical Relevance and Future Direction}

Computational fluid dynamics has increasingly driven in recent years clinical decision-making, surgical planning, and the evaluation of innovative surgical techniques by improving our understanding of congenital heart disease hemodynamics. Considering the excess of morbidity and mortality associated with CHD, and the impact of early detection and appropriate treatment in survival rates, CFD could be routinely used in the clinical management of these patients. Shear stress parameters have been linked to atherosclerosis and aneurysms, and therefore many studies have tried to evaluate the correlation between hemodynamic parameters and disease development. Endothelial cells line the vessel wall and remodel in response to variations in wall shear stress. Specifically, decreased and increased levels of WSS result in an increase in the production of endothelin-1 (ET-1) and nitric oxide (NO), respectively ${ }^{11}$. ET-1 is a potent vasoconstrictor and NO is a potent vasodilator, which they in turn regulate the proliferation of smooth muscle cells and collagen synthesis ${ }^{11}$. In addition, mixing of flow, which is found in curved and tortuous arteries, creates a beneficial environment correlated with the prevention of thrombus formation ${ }^{36}$. In the recent publication by the authors, the measurement of the mean pressure ratios in the pulmonary bifurcation, and flow splits between the left and right pulmonary branches, are hemodynamic markers proposed for the assessment of left pulmonary stenosis, right ventricular dysfunction and pulmonary valve replacement ${ }^{7}$. CFD tools can also be utilized for the design of medical devices, including mechanical heart valves, stents and grafts.

Placement of such devices alters the hemodynamic environment, and it is of immense importance to understand such changes and ensure the durability and proper function of them when surgically placed in patients ${ }^{11}$. A surgical planning tool named Advanced Surgical Planning Interactive Research Environment (ASPIRE) was developed in 1998 in order to help clinicians assess different surgical plans ${ }^{37}$, and similar techniques have since applied this methodology in order to evaluate various surgical procedures ${ }^{38,39}$. Similarly, HeartFlow (HeartFlow Inc., CA) ${ }^{40}$, a diagnostic tool which utilizes CFD to provide cardiologists with a hemodynamic model of the coronary arteries, facilitates customized treatment planning.

Looking towards the future, CFD modelling applied to 
the study of congenital heart disease should have patient outcomes at the forefront. In order to translate these methods into the clinical practice, evidence of CFD-driven treatment and patient outcomes across a variety of ages, gender, and cardiovascular conditions are essential. If these methods are to be used alongside traditional Doppler echocardiography and magnetic resonance imaging, effort should also be directed towards making these methods cost- and time-efficient as well as user-friendly.

\section{Acknowledgements/Funding}

This work was supported in part by the UK Research and Innovation (UKRI) Engineering and Physical Sciences Research Council (EPSRC) through the University of Strathclyde Research Studentship Scheme (SRSS) Student Excellence Awards (SEA) Project No 1619, and Research Excellence Awards (REA), Project No. 1208, by the UKRI Natural Environment Research Council (NERC) Award Ref. NE/T014113/1, and by the European Union's Horizon 2020 research and innovation programme under the Marie Sklodowska-Curie grant agreement No 749185. The MR images in Figure 1 were provided by John Radcliffe Hospital, Oxford [13].

\section{References}

1. van der Linde D, Konings EE, Slager MA, et al. Birth prevalence of congenital heart disease worldwide: a systematic review and meta-analysis. J Am Coll Cardiol. 2011 Nov 15;58(21):2241-7. doi: 10.1016/j.jacc.2011.08.025

2. Mozaffarian D, Benjamin EJ, Go AS, et al. Heart disease and stroke statistics-2015

3. update: a report from the American Heart Association. Circulation. 2015; 131:e29-e322.

4. Wang T, Chen L, Yang T, et al. Congenital Heart Disease and Risk of Cardiovascular

5. Disease: A Meta-Analysis of Cohort Studies. J Am Heart Assoc. 2019;8(10):e012030.

6. Oster ME, Riser AP, Andrews JG, et al. Comorbidities Among Young Adults with Congenital Heart Defects: Results from the Congenital Heart Survey To Recognize Outcomes, Needs, and well-beinG Arizona, Arkansas, and Metropolitan Atlanta, 2016-2019. MMWR Morb Mortal Wkly Rep. 2021 Feb 12;70(6):197-201. doi: 10.15585/ mmwr.mm7006a3.

7. Virani SS, Alonso A, Aparicio HJ, et al. American Heart Association Council on Epidemiology and Prevention Statistics Committee and Stroke Statistics Subcommittee. Heart Disease and Stroke Statistics-2021 Update: A Report From the American Heart Association. Circulation. 2021;143(8):e254-e743.

8. Rao BNS, Anderson RC, Edwards JE. Anatomic variations in the tetralogy of Fallot. Am Heart J. 1971;81:361-371.

9. Boumpouli M, Danton MHD, Gourlay T, Kazakidi A. Blood flow simulations in the pulmonary bifurcation in relation to adult patients with repaired tetralogy of Fallot. Med Eng Phys. 2020b;85:123-138.

10. Black S, Ritos K, Maclean C, et al. P14 Blood flow analysis of the aortic arch using computational fluid dynamics in a coupled 3D-0D framework. Heart. 2020;106:A10-A11.

11. Capelli C, Sauvage E, Giusti G, et al. Patient-specific simulations for planning treatment in congenital heart disease. Interface Focus. 2018;8(1):20170021.

12. Hyde-Linaker G, Black R, Kazakidi A. P2 Fluid-Structure Interaction simulation of multiple bifurcations in arm under transient boundary conditions due to Flow mediated dilation. Heart. 2020;106:A6.

13. Taylor CA, Figueroa CA. Patient-Specific Modeling of Cardiovascular Mechanics. Annu Rev Biomed Eng. 2009;11:109-134.

14. Kazakidi A. Computational studies of blood flow at arterial branches in relation to the localisation of atherosclerosis. Thesis. Imperial College London. 2008.

15. Lygate CA, Schneider JE, Hulbert $K$, et al. Serial high resolution 3D-MRI after aortic banding in mice: band internalization is a source of variability in the hypertrophic response. Basic Res Cardiol. 2006; 101(8-16).

16. Shi Y, Lawford P, Hose R. Review of zero-D and 1-D models of blood flow in the cardiovascular system. BioMedical Engineering Online. 2011;10(1):33.

17. Van Doormaal MA, Kazakidi A, Wylezinska M, et al. Haemodynamics in the mouse aortic arch computed from MRI-derived velocities at the aortic root. J R Soc Interface. 2012;(9):2834-44.

18. Perktold K, Rappitsch G. Computer simulation of local blood flow and vessel mechanics in a compliant carotid artery bifurcation model. J Biomech. 1995;28(7):845-856

19. Boumpouli M, Danton M, Gourlay T, et al. Hemodynamics in the pulmonary bifurcation in relation to adults with congenital heart disease: effect of branching angle and origin. $6^{\text {th }}$ European Conference on Computational Mechanics-7th European Conference on Computational Fluid Dynamics. 2018;1-12.

20. Johnston L, Allen R, Hall-Barrientos P, et al. P13 Computational haemodynamics in turner syndrome patient-specific aortae with PCMRI obtained boundary conditions. Heart. 2020;106:A10.

21. Girdauskas E, Borger MA, Secknus MA, et al. Is aortopathy in bicuspid aortic valve disease a congenital defect or a result of abnormal hemodynamics? A critical reappraisal of a one-sided argument. Eur J Cardiothorac Surg. 2011;39(6):809-14.

22. Mei S, de Souza Júnior FSN, Kuan MYS, et al. Hemodynamics through the congenitally bicuspid aortic valve: a computational fluid dynamics comparison of opening orifice area and leaflet orientation. Perfusion. 2016;31(8):683-690.

23. Kazakidi A, Sherwin SJ, Weinberg PD. Effect of Reynolds number and flow division on patterns of haemodynamic wall shear stress neas branch points in the descending thoracic aorta. J. R. Soc. Interface. 2009;6:539-548.

24. Kazakidi A, Plata AM, Sherwin SJ, et al. Effect of reverse flow on the pattern of wall shear stress near arterial branches. J. R. Soc. Interface. 2011;8:1594-1603.

25. Arzani A, Dyverfeldt $P$, Ebbers $T$, et al. In vivo validation of numerical prediction for turbulence intensity in an aortic coarctation. Ann Biomed Eng. 2012;40(4):860-70.

26. Menon A, Eddinger TJ, Wang $\mathrm{H}$, et al. Altered hemodynamics, endothelial function, and protein expression occur with aortic coarctation and persist after repair. Am J Physiol Heart Circ Physiol. 2012;303(11):H1304-18.

27. Goubergrits L, Riesenkampff E, Yevtushenko P, et al. MRI-based computational fluid dynamics for diagnosis and treatment prediction: clinical validation study in patients with coarctation of aorta. J Magn Reson Imaging. 2015;41(4):909-16.

28. Ralovich K, Itu L, Vitanovski D, et al. Noninvasive hemodynamic assessment, treatment outcome prediction and follow-up of aortic coarctation from MR imaging. Med Phys. 2015;42(5):2143-56. 
29. Cosentino D, Capelli C, Derrick G, et al. Patient-specific computational models to support interventional procedures: a case study of complex aortic re-coarctation. EuroIntervention. 2015;11(6):669-72.

30. Szopos M, Poussineau N, Maday Y, et al. Computational modeling of blood flow in the aorta--insights into eccentric dilatation of the ascending aorta after surgery for coarctation. J Thorac Cardiovasc Surg. 2014;148(4):1572-82

31. Coogan JS, Chan FP, Taylor CA, et al. Computational fluid dynamic simulations of aortic coarctation comparing the effects of surgicaland stent-based treatments on aortic compliance and ventricular workload. Catheter Cardiovasc Interv. 2011;77(5):680-91.

32. Capuano F, Loke U-H, Balaras E. Blood Flow Dynamics at the Pulmonary Artery Bifurcation. Fluids. 2019;4:1-20.

33. Tang BT, Pickard SS, Chan FP, et al. Wall shear stress is decreased in the pulmonary arteries of patients with pulmonary arterial hypertension An image-based, computational fluid dynamics study. Pulmonary Circulation. 2012;2(4):470-476

34. Zhang W, Liu J, Yan Q, et al. Computational haemodynamic analysis of left pulmonary artery angulation effects on pulmonary blood flow. Interact Cardiovasc Thorac Surg. 2016;23:519-525.

35. Chern MJ, Wu MT, Her SW. Numerical study for blood flow in pulmonary arteries after repair of tetralogy of Fallot. Comput Math Methods Med. 2012.
36. Boumpouli M, Danton M, Gourlay T, et al. Understanding the blood flow in realistic and idealised models of the pulmonary bifurcation. Heart Journal. 2020a;106:A3-A4.

37. Spazzapan M, Sastry P, Dunning J, et al. The Use of Biophysical Flow Models in the Surgical Management of Patients Affected by Chronic Thromboembolic Pulmonary Hypertension. Frontiers in Physiology. 2018;9:1-13

38. Cookson AN, Doorly DJ, Sherwin SJ. Mixing Through Stirring of Steady Flow in Small Amplitude Helical Tubes. Annals of Biomedical Engineering. 2009;37(4):710-721.

39. Taylor CA, Draney MT, Ku JP, et al. Predictive medicine:computational techniques in therapeutic decision-making. Comput Aided Surg. 1999;4(5):231- 279.

40. Bove EL, Leval MR, Migliavacca F, et al. Toward optimal hemodynamics:computer modeling of the Fontan circuit. Pediatr Cardiol. 2007;28(6):477-481.

41. Marsden AL, Bernstein AJ, Reddy VM, et al. Evaluation of a novel Y-shaped extracardiac Fontan baffle using computational fluid dynamics. J Thorac Cardiovasc Surg. 2009;137(2):394-403.

42. Taylor CA, Fonte TA, Min JK. Computational fluid dynamics applied to cardiac computed tomography for noninvasive quantification of fractional flow reserve: scientific basis. J Am Coll Cardiol. 2013; $61(22): 2233-41$ 